International Journal of Applied Mathematical Research, 1 (3) (2012) 268-281 (C)Science Publishing Corporation

www.sciencepubco.com/index.php/IJAMR

\title{
Oscillation Criteria of Third Order Nonlinear Neutral Differential Equations
}

\author{
A.A.Soliman, R.A.Sallam, A.Elbitar, A.M.Hassan \\ Department of Mathematics, Faculty of Science, Benha University, \\ Benha-Kalubia 13518, Egypt \\ Email: a_a_soliman@hotmail.com \\ Department of Mathematics, Faculty of Science, Monoufia University, \\ Shibin EL-Koom , Egypt \\ Email: ragaasallam@yahoo.com \\ Department of Mathematics, Faculty of Science, Benha University, \\ Benha-Kalubia 13518, Egypt \\ Email: ahmed.mohamed@fsc.bu.edu.eg
}

\begin{abstract}
In this paper we will study the criteria for oscillation of the equation

$$
\left[a(t)\left(z^{\prime \prime}(t)\right)^{\gamma}\right]^{\prime}+\sum_{j=1}^{m} f_{j}\left(t, x\left(\tau_{j}(t)\right)\right)=0
$$

and establish new oscillation criteria some examples of the obtained results are given. Our technique is Riccati's method.
\end{abstract}

Keywords: Oscillation, Third order, Nonlinear equation, Neutral type.

\section{Introduction}

Consider the couple of third-order neutral differential equation of the form

$$
\left[a(t)\left(z^{\prime \prime}(t)\right)^{\gamma}\right]^{\prime}+\sum_{j=1}^{m} f_{j}\left(t, x\left(\tau_{j}(t)\right)\right)=0
$$

where $z(t)=x(t) \pm \sum_{i=1}^{n} p_{i}(t) x\left(\sigma_{i}(t)\right)$, under the assumptions. 
$\left(I_{1}\right) a(t) \in C\left(\left[t_{0}, \infty\right),(0, \infty)\right), \gamma>0, \int_{t_{0}}^{\infty} a(s)^{\frac{-1}{\gamma}} d s=\infty$

$\left(I_{2}\right) p_{i}(t) \in C\left(\left[t_{0}, \infty\right), R\right),-\mu \leq p_{i}(t) \leq 1 \quad$ for all $i=1,2,3, \ldots, n ; \mu \in(0,1)$;

$\left(I_{3}\right) \sigma_{i}(t) \in C\left(\left[t_{0}, \infty\right), R\right), \sigma_{i}(t) \leq t ; \lim _{t \rightarrow \infty} \sigma_{i}(t)=\infty$ for all $i=1,2,3, \ldots, n$;

$\left(I_{4}\right) \tau_{j}(t) \in C\left(\left[t_{0}, \infty\right), R\right), \tau_{j}(t) \leq t ; \lim _{t \rightarrow \infty} \tau_{j}(t)=\infty$ for all $j=1,2,3, \ldots, m$;

( $\left.I_{5}\right) f_{j}\left(t, x\left(\tau_{j}(t)\right)\right) \in C\left(\left[t_{0}, \infty\right) \times R, R\right)$ and there exists $q_{j}(t) \in C\left(\left[t_{0}, \infty\right),(0, \infty)\right)$ such that $f_{j}\left(t, x\left(\tau_{j}(t)\right)\right)$ sign $x \geq q_{j}(t)\left|x^{\gamma}\left(\tau_{j}(t)\right)\right|^{\gamma}$.

Neutral differential equations find numerous applications in natural science and technology. For instance, they are frequently used for the study of distributed networks containing lossless transmission lines;see[1-15].

In the last decades, there are many studies that have been made on the oscillatory behavior of solutions of second and first differential equations. For the third-order equations we have greatly fewer results than first and second-order equations;

For instance, B.Baculikova, J.Dzurina [4], examined the oscillation of the thirdorder nonlinear differential equations

$$
\left[a(t)\left(x^{\prime \prime}(t)\right)^{\gamma}\right]^{\prime}+q(t) f(x(\tau(t)))=0,
$$

where $a(t), q(t)$ are positive and $\gamma$ is a quotient of odd positive integers, B.Baculikova, J.Dzurina [3] studied the oscillation of the third-order nonlinear neutral differential equations

$$
\left[a(t)\left([x(t) \pm p(t) x(\delta(t))]^{\prime \prime}\right)^{\gamma}\right]^{\prime}+q(t) x^{\gamma}(\tau(t))=0,
$$

where $\mathrm{a}(\mathrm{t}), \mathrm{q}(\mathrm{t})$ and $\mathrm{p}(\mathrm{t})$ are positive and $\gamma$ is a quotient of odd positive integers,

B.Baculikova, E.M.Elabbasy, S.H.Saker, J.Dzurina [2] considered the thirdorder equation

$$
\left[b(t)\left(\left(a(t) x^{\prime}(t)^{\prime}\right)^{\gamma}\right]^{\prime}+q(t) x^{\gamma}(\tau(t))=0,\right.
$$

where $b(t), a(t)$ and $q(t)$ are positive and $\gamma$ is a quotient of odd positive integers.

In this paper we will improve and extend some of main results of [6]. we will use the technique in [1] to obtain criteria for oscillation of third-order neutral delay differential equations.

For simplicity we define

$A(t)=\int_{t_{0}}^{\infty} a(s)^{\frac{-1}{\gamma}} d s ; \quad \widehat{Q}(t)=\int_{t}^{\infty} \sum_{j=1}^{m} q_{j}(s)\left[1-\sum_{i=1}^{n} p_{i}(t)\left(\tau_{j}(s)\right)\right]^{\gamma} d s ;$

$R(t)=\gamma \tau_{j}^{\prime}(t) A\left[\tau_{j}(t)\right] ; \quad Q(t)=\int_{t}^{\infty} \sum_{j=1}^{m} q_{j}(s) d s$ 


\section{Main Results}

\subsection{Oscillation criteria for $0 \leq p_{i}(t) \leq 1$ for all $i=1,2,3, \ldots, n$.}

In this part we will study the criteria for

$$
\left[a(t)\left[\left(x(t)+\sum_{i=1}^{n} p_{i}(t) x\left(\sigma_{i}(t)\right)\right)^{\prime \prime}\right]^{\gamma}\right]^{\prime}+\sum_{j=1}^{m} f_{j}\left(t, x\left(\tau_{j}(t)\right)\right)=0 .
$$

The following lemma, we will be needed in the sequel .

Lemma 2.1. Let $x(t)$ be a positive solution of $E q(2)$, then there are only the following two cases for $z(t)$ :

1. $z(t)>0, z^{\prime}(t)>0, z^{\prime \prime}(t)>0$;

2. $z(t)>0, z^{\prime}(t)<0, z^{\prime \prime}(t)>0$;

for $t \geq t_{1}$, where $t_{1}$ is sufficiently large.

Proof. Assume that $x(t)$ be a positive solution of $\operatorname{Eq}(2)$ on $\left[t_{0}, \infty\right)$ we see that $z(t)>0, x(t)>0$

From $\left(I_{5}\right), \operatorname{Eq}(2)$ we have

$$
\begin{aligned}
{\left[a(t)\left(z^{\prime \prime}(t)\right)^{\gamma}\right]^{\prime} } & =-\sum_{j=1}^{m} f_{j}\left(t, x\left(\tau_{j}(t)\right)\right) \\
& \leq-\sum_{j=1}^{m} x^{\gamma}\left(\tau_{j}(t)\right) \\
& <0
\end{aligned}
$$

Thus $\left[a(t)\left(z^{\prime \prime}(t)\right)^{\gamma}\right]$ is nonincreasing and of one sign. There for $z^{\prime \prime}(t)$ is also of one sign and so we have two cases: $z^{\prime \prime}(t)<0$ or $z^{\prime \prime}(t)>0$ for all $t \geq t_{1}$. If we admit that $z^{\prime \prime}(t)<0$.then there exist a constant $M>0$ such that

$$
a(t)\left(z^{\prime \prime}(t)\right)^{\gamma} \leq-M<0
$$

integrating on $\left[t_{1}, t\right]$, we obtain

$$
z^{\prime}(t) \leq z^{\prime}\left(t_{1}\right)-M^{\frac{1}{\gamma}} \int_{t_{1}}^{t} a^{\frac{-1}{\gamma}}(s) d s
$$

as $t \rightarrow \infty$, we get $z^{\prime}(t) \rightarrow-\infty$.thus $z^{\prime}(t)<0$ eventually. But $z^{\prime \prime}(t)<0$ and $z^{\prime}<0$ eventually imply $z(t)<0$ for $t \leq t_{1}$. This contradiction, and this proves that $z^{\prime \prime}(t)>0$. 
Theorem 2.2. Suppose that Eq(Q) is nonoscillatory, then there exist a positive function $V(t)$ on $[T, \infty)$. such that

1.

$$
\widehat{Q}(t)<\infty, \quad \int_{t}^{\infty} R(s) \widehat{Q}^{\frac{\gamma+1}{\gamma}}(s) d s<\infty
$$

2.

$$
V(t) \geq \widehat{Q}(t)+\int_{t}^{\infty} R(s) V^{\frac{\gamma+1}{\gamma}}(s) d s \text { for all } t \geq T \geq t_{0}
$$

3.

$$
\limsup _{t \rightarrow \infty} V(t)\left[\int_{t_{0}}^{\tau_{j}(t)} \tau_{j}^{\prime}(s) A\left[\tau_{j}(s)\right] d s\right]^{\gamma} \leq 1
$$

Proof. Let $x(t)$ be nonoscillatory solution of $\mathrm{Eq}(2)$. Assume that $x(t)>0$, $x\left(\sigma_{i}(t)\right)>0$ for all $i=1,2, \ldots, n ; x\left(\tau_{j}(t)\right)>0$ for all $j=1,2, \ldots, m$ then $z(t)>0, z(t)>x(t) . \operatorname{from}\left(I_{5}\right)$

$$
\begin{aligned}
{\left[a(t)\left(z^{\prime \prime}(t)\right)^{\gamma}\right]^{\prime} } & =-\sum_{j=1}^{m} f_{j}\left(t, x\left(\tau_{j}(t)\right)\right) \\
& \leq-\sum_{j=1}^{m} q_{j}(t) x^{\gamma}\left(\tau_{j}(t)\right)
\end{aligned}
$$

since;

$$
\begin{aligned}
x(t) & =z(t)-\sum_{i=1}^{n} p_{i}(t) x\left(\sigma_{i}(t)\right), \\
x\left(\tau_{j}(t)\right) & =z\left(\tau_{j}(t)\right)-\sum_{i=1}^{n} p_{i}\left(\tau_{j}(t)\right) x\left(\sigma_{i}\left(\tau_{j}(t)\right)\right), \\
& \geq z\left(\tau_{j}(t)\right)-\sum_{i=1}^{n} p_{i}\left(\tau_{j}(t)\right) z\left(\sigma_{i}\left(\tau_{j}(t)\right)\right), \\
& \geq\left[1-\sum_{i=1}^{n} p_{i}\left(\tau_{j}(t)\right)\right] z\left(\tau_{j}(t)\right),
\end{aligned}
$$

$\operatorname{from}(6),(7)$

$$
\frac{\left[a(t)\left(z^{\prime \prime}(t)\right)^{\gamma}\right]^{\prime}}{z^{\gamma}\left(\tau_{j}(t)\right)} \leq-\sum_{j=1}^{m} q_{j}(t)\left[1-\sum_{i=1}^{n} p_{i}\left(\tau_{j}(t)\right)\right]^{\gamma} .
$$

Now, we define function $V(t)$

$$
V(t)=\frac{\left[a(t)\left(z^{\prime \prime}(t)\right)^{\gamma}\right]}{z^{\gamma}\left(\tau_{j}(t)\right)}
$$




$$
\begin{aligned}
V^{\prime}(t) & =\frac{\left[a(t)\left(z^{\prime \prime}(t)\right)^{\gamma}\right]^{\prime}}{z^{\gamma}\left(\tau_{j}(t)\right)}-\gamma \frac{\left[a(t)\left(z^{\prime \prime}(t)\right)^{\gamma}\right] z^{\prime}\left(\tau_{j}(t)\right)\left(\tau_{j}^{\prime}(t)\right)}{z^{\gamma+1}\left(\tau_{j}(t)\right)} \\
& \leq-\sum_{j=1}^{m} q_{j}(t)\left[1-\sum_{i=1}^{n} p_{i}\left(\tau_{j}(t)\right)\right]^{\gamma}-\gamma V(t) \frac{z^{\prime}\left(\tau_{j}(t)\right)\left(\tau_{j}^{\prime}(t)\right)}{z\left(\tau_{j}(t)\right)}
\end{aligned}
$$

since

$$
z^{\prime}(t) \geq \int_{t_{0}}^{t} z^{\prime \prime}(s) d s=\int_{t_{0}}^{t} a^{\frac{-1}{\gamma}}(s)\left[a(s)\left(z^{\prime \prime}(s)\right)^{\gamma}\right]^{\frac{1}{\gamma}} d s,
$$

from $\left(I_{1}\right)$ and $\left(I_{5}\right)$, we get

$$
\begin{aligned}
z^{\prime}(t) & \geq\left[a(t)\left(z^{\prime \prime}(t)\right)^{\gamma}\right]^{\frac{1}{\gamma}} \int_{t_{0}}^{t} a^{\frac{-1}{\gamma}}(s) d s \\
z^{\prime}\left(\tau_{j}(t)\right) & \geq\left[a\left(\tau_{j}(t)\right)\left(z^{\prime \prime}\left(\tau_{j}(t)\right)\right)^{\gamma}\right]^{\frac{1}{\gamma}} \int_{t_{0}}^{\tau_{j}(t)} a^{\frac{-1}{\gamma}}(s) d s, \\
z^{\prime}\left(\tau_{j}(t)\right) & \geq\left[a\left(\tau_{j}(t)\right)\left(z^{\prime \prime}\left(\tau_{j}(t)\right)\right)^{\gamma}\right]^{\frac{1}{\gamma}} A\left[\tau_{j}(t)\right],
\end{aligned}
$$

from $(2),\left(I_{1}\right),\left(I_{4}\right)$ and $\left(I_{5}\right)$, we get

$$
\left[a(t)\left(z^{\prime \prime}(t)\right)^{\gamma}\right] \leq\left[a\left(\tau_{j}(t)\right)\left(z^{\prime \prime}\left(\tau_{j}(t)\right)\right)^{\gamma}\right],
$$

from (9), (10)

$$
z^{\prime}\left(\tau_{j}(t)\right) \geq\left[a(t)\left(z^{\prime \prime}(t)\right)^{\gamma}\right]^{\frac{1}{\gamma}} A\left[\tau_{j}(t)\right]
$$

from (8) and (11), we have

$$
\begin{aligned}
& V^{\prime}(t) \leq-\sum_{j=1}^{m} q_{j}(t)\left[1-\sum_{i=1}^{n} p_{i}\left(\tau_{j}(t)\right)\right]^{\gamma}-\gamma V(t) \frac{\left[a(t)\left(z^{\prime \prime}(t)\right)^{\gamma}\right]^{\frac{1}{\gamma}} A\left[\tau_{j}(t)\right]\left(\tau_{j}^{\prime}(t)\right)}{z\left(\tau_{j}(t)\right)} \\
& \leq-\sum_{j=1}^{m} q_{j}(t)\left[1-\sum_{i=1}^{n} p_{i}\left(\tau_{j}(t)\right)\right]^{\gamma}-\gamma V^{\frac{\gamma+1}{\gamma}}(t) A\left[\tau_{j}(t)\right]\left(\tau_{j}^{\prime}(t)\right) \\
& V^{\prime}(t)+\sum_{j=1}^{m} q_{j}(t)\left[1-\sum_{i=1}^{n} p_{i}\left(\tau_{j}(t)\right)\right]^{\gamma}+R(t) V^{\frac{\gamma+1}{\gamma}}(t)
\end{aligned}
$$

integrating on $\left[t, t^{\prime}\right]$

$$
V\left(t^{\prime}\right)-V(t)+\int_{t}^{t^{\prime}} \sum_{j=1}^{m} q_{j}(s)\left[1-\sum_{i=1}^{n} p_{i}\left(\tau_{j}(s)\right)\right]^{\gamma} d s+\int_{t}^{t^{\prime}} R(s) V^{\frac{\gamma+1}{\gamma}}(s) d s \leq 0,
$$

assume that $\widehat{Q}(t)<\infty$ for all $t \geq t_{2}$, otherwise from the above inequality

$$
V\left(t^{\prime}\right) \leq V(t)-\int_{t}^{t^{\prime}} \sum_{j=1}^{m} q_{j}(s)\left[1-\sum_{i=1}^{n} p_{i}\left(\tau_{j}(s)\right)\right]^{\gamma} d s
$$


this lead to $V\left(t^{\prime}\right) \rightarrow-\infty$ as $t^{\prime} \rightarrow \infty$. Which contradiction $(V(t)$ positive $)$, Similarly we can show that

$$
\int_{t}^{\infty} R(s) V^{\frac{\gamma+1}{\gamma}}(s) d s<0 .
$$

To prove (4) from Theorem 2.2

from (12) we define $\lim _{t \rightarrow \infty} V(t)=V^{*}$, from (15)letting $t^{\prime} \rightarrow \infty$ in (14)

$$
V(t) \geq \widehat{Q}(t)+\int_{t}^{\infty} R(s) V^{\frac{\gamma+1}{\gamma}}(s) d s \text { for all } t \geq T \geq t_{0}
$$

To prove (5)

from $(13)$ and $\sum_{j=1}^{m} q_{j}(t)\left[1-\sum_{i=1}^{n} p_{i}\left(\tau_{j}(t)\right)\right]^{\gamma}>0$, then

$$
V^{\prime}(t)+R(t) V^{\frac{\gamma+1}{\gamma}}(t)<0
$$

since $V(t)>0$ and $R(t)>0$, then from $(16), V^{\prime}(t)<0$, and

$$
\begin{gathered}
-\frac{V^{\prime}(t)}{\gamma V^{\frac{\gamma+1}{\gamma}}(t)}>A\left[\tau_{j}(t)\right]\left(\tau_{j}^{\prime}(t)\right) \\
\left(V^{\frac{-1}{\gamma}(t)}\right)^{\prime}>A\left[\tau_{j}(t)\right]\left(\tau_{j}^{\prime}(t)\right) .
\end{gathered}
$$

Integrating the above inequality on $\left[t_{0}, \tau_{j}(t)\right]$, yields

$$
\begin{aligned}
V^{\frac{-1}{\gamma}}(t) & >\int_{t_{0}}^{\tau_{j}(t)} A\left[\tau_{j}(s)\right]\left(\tau_{j}^{\prime}(s)\right) d s \\
\frac{1}{V} & >\left[\int_{t_{0}}^{\tau_{j}(t)} A\left[\tau_{j}(s)\right]\left(\tau_{j}^{\prime}(s)\right) d s\right]^{\gamma} .
\end{aligned}
$$

This complete proof of Theorem 2.2

Corollary 2.3. Assume that

$$
\liminf _{t \rightarrow \infty} \frac{1}{\widehat{Q}(t)} \int_{t}^{\infty} \widehat{Q}^{\frac{\gamma+1}{\gamma}}(s) R(s) d s>\frac{\gamma}{(\gamma+1)^{\frac{\gamma+1}{\gamma}}} .
$$

Then Eq.(2) is oscillatory.

Proof. Suppose the contrary that $\mathrm{Eq}(2)$ is nonoscillatory from theorem (4) we find

$$
V(t) \geq \widehat{Q}(t)+\int_{t}^{\infty} R(s) V^{\frac{\gamma+1}{\gamma}}(s) d s
$$


and from the assumption of the corollary .there exist $\beta>\frac{\gamma}{(\gamma+1)^{\frac{\gamma+1}{\gamma}}}$ such that

$$
\liminf _{t \rightarrow \infty} \frac{1}{\widehat{Q}(t)} \int_{t}^{\infty} \widehat{Q}^{\frac{\gamma+1}{\gamma}}(s) R(s) d s>\beta
$$

$\operatorname{from}(4)$

$$
\begin{gathered}
\frac{V(t)}{\widehat{Q}(t)} \geq 1+\frac{1}{\widehat{Q}(t)} \int_{t}^{\infty} R(s) V^{\frac{\gamma+1}{\gamma}}(s) d s \\
\frac{V(t)}{\widehat{Q}(t)} \geq 1+\frac{1}{\widehat{Q}(t)} \int_{t}^{\infty} R(s) V^{\frac{\gamma+1}{\gamma}}(s) d s \\
\geq 1+\frac{1}{\widehat{Q}(t)} \int_{t}^{\infty} R(s) \widehat{Q}^{\frac{\gamma+1}{\gamma}}(s)\left(\frac{V(s)}{\widehat{Q}(s)}\right)^{\frac{\gamma+1}{\gamma}} d s .
\end{gathered}
$$

Let $\lambda=\inf _{t \geq T}\left(\frac{V(t)}{\widehat{Q}(t)}\right)$, then $\lambda \geq 1$ and $\lambda \geq 1+\beta \lambda^{\frac{\gamma+1}{\gamma}}$ by simple calculation we get $\lambda-\beta \lambda^{\frac{\gamma+1}{\gamma}} \leq \frac{\gamma^{\gamma}}{(\gamma+1)^{\gamma}} \frac{1}{\beta^{\gamma}}$ a contradiction, $\beta=\frac{\gamma^{\gamma}}{(\gamma+1)^{\frac{\gamma+1}{\gamma}}}$ this contradiction lead to $\operatorname{Eq}(2)$ is oscillatory.

Following [1],Let $\left\{y_{n}(t)\right\}_{n=0}^{\infty}$ be a sequence of continuous functions defined as follows (if they exist):

$y_{0}(t)=\widehat{Q}(t)$ for all $t \geq t_{0}$ and

$$
y_{n}(t)=\int_{t}^{\infty} R(s) y_{n-1}^{\frac{\gamma+1}{\gamma}}(s) d s+\widehat{Q}(t)
$$

Lemma 2.4. [1]. If $E q\left(\right.$ 2) is nonoscillatory, then $y_{n}(t) \leq V(t)$ where $V(t)$ be as defined in Theorem 2.2 and there exists a positive function $y(t)$ on $[t, \infty)$, such that $\lim _{t \rightarrow \infty} y_{n}(t)=y$ for $t \geq T \geq t_{0}$. In addition we have

$$
y(t)=\int_{t}^{\infty} R(s) y^{\frac{\gamma+1}{\gamma}}(s) d s+\widehat{Q}(t) .
$$

Proof. suppose that $\mathrm{Eq}(2)$ is nonoscillatory . from (Theorem 2.2) $y_{0}(t) \leq$ $y_{1}(t)$, by induction $y_{n}(t) \leq y_{n+1}(t)$ for $n=0,1,2, \ldots$.

By the other hand from $(3)$, we have $V(t) \geq \widehat{Q}(t)=y_{0}(t)$.

Inductively, we get $V(t) \geq y_{n}(t)$ for $n=0,1,2, \ldots$.

Thus, the sequence $\left\{y_{n}(t)\right\}_{n=0}^{\infty}$ converges to $y(t)$ on $[T, \infty)$.by Lebesgue's monotone convergence theorem, and letting $n \rightarrow \infty$ in Eq (18)we get (19).

Corollary 2.5. Let $y_{n}(t)$ defined by (18).if there exists some $y_{n}(t)$ such that

$$
\limsup _{t \rightarrow \infty} y_{n}(t)\left[\int_{t_{0}}^{\tau_{j}(t)} \tau_{j}^{\prime}(s) A\left[\tau_{j}(s)\right] d s\right]^{\gamma}>1 \text { for all } n=0,1,2, \ldots \text {. }
$$

Then $E q(2)$ is oscillatory. 
Proof. Suppose that $\mathrm{Eq}(2)$ is nonoscillatory, then from Theorem 2.2

$$
\limsup _{t \rightarrow \infty} V(t)\left[\int_{t_{0}}^{\tau_{j}(t)} \tau_{j}^{\prime}(s) A\left[\tau_{j}(s)\right] d s\right]^{\gamma} \leq 1
$$

by contrary and from Lemma 2.4 we get

$$
\limsup _{t \rightarrow \infty} y_{n}(t)\left[\int_{t_{0}}^{\tau_{j}(t)} \tau_{j}^{\prime}(s) A\left[\tau_{j}(s)\right] d s\right]^{\gamma}>1
$$

This lead to $\operatorname{Eq}(2)$ is oscillatory.

Corollary 2.6. Assume that

$$
\limsup _{t \rightarrow \infty}\left[\int_{t_{0}}^{\tau_{j}(t)} \tau_{j}^{\prime}(s) A\left[\tau_{j}(s)\right] d s\right]^{\gamma}\left[\int_{t}^{\infty} R(s) \widehat{Q}^{\frac{\gamma+1}{\gamma}}(s) d s+\widehat{Q}(t)\right]>1
$$

Then $E q(2)$ is oscillatory.

\subsection{Oscillation criteria for $-\mu \leq \sum_{i=1}^{n} p_{i}(t) \leq 0$.}

In this section we will study the criteria for

$$
\left[a(t)\left[\left(x(t)-\sum_{i=1}^{n} p_{i}(t) x\left(\sigma_{i}(t)\right)\right)^{\prime \prime}\right]^{\gamma}\right]^{\prime}+\sum_{j=1}^{m} f_{j}\left(t, x\left(\tau_{j}(t)\right)\right)=0,
$$

Theorem 2.7. Assume that every solution of Eq(21) is neither osillatory nor tends to zero .then there exists a positive function $V(t)$ on $[T, \infty)$ such that

1 .

$$
Q(t)<\infty \quad, \quad \int_{t}^{\infty} R(s) Q^{\frac{\gamma+1}{\gamma}}(s) d s<\infty
$$

2.

$$
V(t) \geq Q(t)+\int_{t}^{\infty} R(s) V^{\frac{\gamma+1}{\gamma}}(s) d s \text { for all } t \geq T \geq t_{0}
$$

3.

$$
\limsup _{t \rightarrow \infty} V(t)\left[\int_{t_{0}}^{\tau_{j}(t)} \tau_{j}^{\prime}(s) A\left[\tau_{j}(s)\right] d s\right]^{\gamma} \leq 1
$$


Proof. Let $x(t)$ be a solution which is neither oscillatory nor tends to zero such that $x(t)>0 ; x\left(\sigma_{i}(t)\right)>0 ; x\left(\tau_{j}(t)\right)>0$

from (6) we have

$$
\begin{aligned}
{\left[a(t)\left(z^{\prime \prime}(t)\right)^{\gamma}\right]^{\prime} } & =-\sum_{j=1}^{m} f_{j}\left(t, x\left(\tau_{j}(t)\right)\right) \\
& \leq-\sum_{j=1}^{m} q_{j}(t) x^{\gamma}\left(\tau_{j}(t)\right),
\end{aligned}
$$

we have two possible cases for $z(t)$

$$
\begin{aligned}
& \text { I } z(t)>0, \\
& \text { II } z(t)<0,
\end{aligned}
$$

(I) $z(t)>0$, then proof will be as proof of theorem 2.2 but $\widehat{Q}(t)$ replaced by $Q(t)$ (II) $z(t)<0$ eventually for all $t \geq t_{2} \geq t_{1} \geq t_{0}$, then we have two cases for $x(t)$

(a) $x(t)$ is unbounded

(b) $x(t)$ is bounded

(a) $x(t)$ is unbounded

Assume that $x(t)$ is unbounded, then

$$
x(t)=z(t)-\sum_{i=1}^{n} p_{i}(t) x\left(\sigma_{i}(t)\right)<-\sum_{i=1}^{n} p_{i}(t) x\left(\sigma_{i}(t)\right)<\sum_{i=1}^{n} x\left(\sigma_{i}(t)\right) .
$$

Since $x(t)$ is unbounded, then we can choose a sequence $\left\{T_{n}\right\}_{n=1}^{\infty}$ satisfying $\lim _{n \rightarrow \infty} T_{n}=\infty$ from which $\lim _{n \rightarrow \infty} x\left(T_{n}\right)=\infty$ and $\max _{x} x(t)=x\left(T_{n}\right)$ by choosing $N$ large such that $\sigma_{i}\left(T_{N}\right)>T_{1}$ for all $T_{N}>t_{2}$. Thus $\max x(t)=$ $x\left(T_{N}\right)$. this contradict with $(25)$.

(b) $x(t)$ is bounded

Suppose that $x(t)$ is bounded, we show that $x(t) \rightarrow 0$ as $t \rightarrow \infty$ since

$$
\limsup _{t \rightarrow \infty}(z(t)) \leq 0
$$

then we have 


$$
\begin{array}{r}
\limsup _{t \rightarrow \infty}\left(x(t)+\sum_{i=1}^{n} p_{i}(t) x\left(\sigma_{i}(t)\right) \leq 0\right. \\
\limsup _{t \rightarrow \infty} x(t)+\limsup _{t \rightarrow \infty} x(t) \sum_{i=1}^{n} p_{i}(t) x\left(\sigma_{i}(t)\right) \leq 0 \\
(1-\mu) \limsup _{t \rightarrow \infty} x(t) \leq 0 .
\end{array}
$$

This shows that $x(t) \rightarrow 0$ as $t \rightarrow \infty$.

Corollary 2.8. Assume that

$$
\limsup _{t \rightarrow \infty}\left[\int_{t_{0}}^{\tau_{j}(t)} \tau_{j}^{\prime}(s) A\left[\tau_{j}(s)\right] d s\right]^{\gamma}\left[\int_{t}^{\infty} R(s) Q^{\frac{\gamma+1}{\gamma}}(s) d s+Q(t)\right]>1 .
$$

Then every solution of $E q(21)$ is either oscillatory or tends to zero.

Corollary 2.9. Assume that

$$
\liminf _{t \rightarrow \infty} \frac{1}{Q(t)} \int_{t}^{\infty} Q^{\frac{\gamma+1}{\gamma}}(s) R(s) d s>\frac{\gamma}{(\gamma+1)^{\frac{\gamma+1}{\gamma}}} .
$$

Then every solution of Eq(21) is either oscillatory or tends to zero.

Remark 2.10. We can get oscillation criteria of the equation

$$
\left[a(t)\left(z^{\prime \prime}(t)\right)^{\gamma}\right]^{\prime}+f(t, x(\tau(t)))=0,
$$

where $z(t)=x(t) \pm p(t) x(\sigma(t))$, if we put $j=i=1$.

\section{$3 \quad$ Examples}

Example 1. Consider

$$
\left[t\left(z^{\prime \prime}(t)\right)^{3}\right]^{\prime}+\sum_{j=1}^{2} f_{j}\left(t, x\left(\tau_{j}(t)\right)\right)=0, \quad t \geq 0
$$

$z(t)=x(t)+\sum_{i=1}^{2} p_{i}(t) x\left(\sigma_{i}(t)\right), p_{1}(t)=\frac{1}{2}, p_{2}(t)=\frac{1}{4}, q_{1}(t)=\frac{2 a}{t^{6}}, q_{2}(t)=\frac{4 a}{t^{6}}$, $a>0, \tau_{1}(t)=\frac{t}{2}, \tau_{2}(t)=\frac{t}{3}$.

It is clear that

$a(t)=t, \quad \int_{t}^{\infty} a^{\frac{-1}{\gamma}}(s) d s=\int_{t}^{\infty} s^{\frac{-1}{3}} d s=\infty$,

$0 \leq p_{1}(t), p_{2}(t) \leq 1 ; \quad q_{1}(t), q_{2}(t)$ positive.

since 


$$
\begin{gathered}
\widehat{Q}(t)=\int_{t}^{\infty} \sum_{j=1}^{m} q_{j}(s)\left[1-\sum_{i=1}^{n} p_{i}(t)\left(\tau_{j}(s)\right)\right]^{\gamma} d s \\
\widehat{Q}(t)=\int_{t}^{\infty}\left[q_{1}(s)\left(1-\left(p_{1}(s)+p_{2}(s)\right)\right]^{3}+\left[q_{2}(s)\left(1-\left(p_{1}(s)+p_{2}(s)\right)\right)\right]^{3} d s\right. \\
=\left(\frac{1}{4}\right)^{3} \int_{t}^{\infty} \frac{6 a}{s^{6}} d s \\
=\left(\frac{1}{4}\right)^{3}\left(\frac{6 a}{5}\right)\left(\frac{1}{t^{5}}\right) . \\
R(t)=\gamma \tau_{j}^{\prime}(t) A\left[\tau_{j}(t)\right] \\
=3\left[\tau_{1}^{\prime}(t) A\left[\tau_{1}(t)\right]+\tau_{2}^{\prime}(t) A\left[\tau_{2}(t)\right]\right] \\
=\left(\frac{9}{2}\right)\left[\left(\frac{1}{2}\right)^{5 / 3}+\left(\frac{1}{3}\right)^{5 / 3}\right] t^{2 / 3} .
\end{gathered}
$$

from (17)

$\liminf _{t \rightarrow \infty} \frac{1}{\widehat{Q}(t)} \int_{t}^{\infty} \widehat{Q}^{\frac{\gamma+1}{\gamma}}(s) R(s) d s=\left(\frac{9}{10}\right)\left(\frac{1}{4}\right)\left(\frac{6 a}{5}\right)^{1 / 3}\left[\left(\frac{1}{2}\right)^{5 / 3}+\left(\frac{1}{3}\right)^{5 / 3}\right]$.

since $\frac{\gamma}{(\gamma+1)^{\gamma}}=\frac{3}{(4)^{4 / 3}}$

Then the solution of (28)is oscillatory when $a>71.89238009$.

Example 2. Consider

$$
\left[t\left(z^{\prime \prime}(t)\right)^{3}\right]^{\prime}+\sum_{j=1}^{2} f_{j}\left(t, x\left(\tau_{j}(t)\right)\right)=0, \quad t \geq 0
$$

$z(t)=x(t)+\sum_{i=1}^{2} p_{i}(t) x\left(\sigma_{i}(t)\right), p_{1}(t)=\frac{-1}{2}, p_{2}(t)=\frac{-1}{4}, q_{1}(t)=\frac{2 a}{t^{6}}, q_{2}(t)=\frac{4 a}{t^{6}}$, $a>0, \tau_{1}(t)=\frac{t}{2}, \tau_{2}(t)=\frac{t}{3}$.

It is clear that

$a(t)=t, \quad \int_{t}^{\infty} a^{\frac{-1}{\gamma}}(s) d s=\int_{t}^{\infty} s^{\frac{-1}{3}} d s=\infty$,

$0 \leq p_{1}(t), p_{2}(t) \leq 1 ; \quad q_{1}(t), q_{2}(t)$ positive.

since

$$
Q(t)=\int_{t}^{\infty} \sum_{j=1}^{m} q_{j}(s) d s=\left(\frac{6 a}{5}\right)\left(\frac{1}{t^{5}}\right)
$$




$$
R(t)=\left(\frac{9}{2}\right)\left[\left(\frac{1}{2}\right)^{5 / 3}+\left(\frac{1}{3}\right)^{5 / 3}\right] t^{2 / 3}
$$

from (27)

$$
\liminf _{t \rightarrow \infty} \frac{1}{Q(t)} \int_{t}^{\infty} Q^{\frac{\gamma+1}{\gamma}}(s) R(s) d s=\left(\frac{9}{10}\right)\left(\frac{6 a}{5}\right)^{1 / 3}\left[\left(\frac{1}{2}\right)^{5 / 3}+\left(\frac{1}{3}\right)^{5 / 3}\right] .
$$

since $\frac{\gamma}{(\gamma+1)^{\gamma}}=\frac{3}{(4)^{4 / 3}}$

Then the solution of (29)is oscillatory or tends to zero when $a>1.123318444$.

Example 3. [13] Consider

$$
\left(\left(x^{\prime \prime}(t)\right)^{3}\right)^{\prime}+\frac{a}{t^{7}} x^{3}(\lambda t)=0 \quad, a>0, \quad 0<\lambda<1, \quad t \geq 1 .
$$

Note that $p(t)=0, q(t)=\frac{a}{t^{7}}, \gamma=3, \tau(t)=\lambda t, a(t)=1, \int_{t}^{\infty} a^{\frac{-1}{\gamma}}(s) d s=\infty$ since

$$
\begin{aligned}
\widehat{Q}(t)=\int_{t}^{\infty} \sum_{j=1}^{m} q_{j}(s)\left[1-\sum_{i=1}^{n} p_{i}(t)\left(\tau_{j}(s)\right)\right]^{\gamma} d s \\
=\int_{t}^{\infty} q(s)[1-p(t)(\tau(s))]^{\gamma} d s \\
=\frac{a}{6 t^{6}} . \\
R(t)=\gamma \tau_{j}^{\prime}(t) A\left[\tau_{j}(t)\right] \\
=\gamma \tau^{\prime}(t) A[\tau(t)] \\
=(3 \lambda)\left(\lambda t-t_{0}\right) .
\end{aligned}
$$

from (17)

$$
\liminf _{t \rightarrow \infty} \frac{1}{\widehat{Q}(t)} \int_{t}^{\infty} \widehat{Q}^{\frac{\gamma+1}{\gamma}}(s) R(s) d s=\frac{3 \lambda^{2}}{6}\left(\frac{a}{6}\right)^{1 / 3}
$$

since $\frac{\gamma}{(\gamma+1)^{\gamma}}=\frac{3}{(4)^{4 / 3}}$

Then Eq(3.3.1)is oscillatory if $a \lambda^{6}>\frac{3^{4}}{4^{2}}$

This result is consistent with the result in Example 168 of [13] . 


\section{References}

[1] R. P. AGARWAL,SHIOW-LING SHIEH AND CHEH-CHIH YEH, Oscillation Criteria for Second-Order Retarded Differential Equations, Math. Comput. Modelling Vol. 26, No. 4, pp. 1-11.

[2] B. Baculkov, E.M. Elabbasy, S.H. Saker, J. Dzurina, Oscillation criteria for third-order nonlinear differential equations, Math. Slovaca 58 (2) (2008) 1-20.

[3] B. Baculkov, J. Dzurina, Oscillation of third-order neutral differential equations, Mathematical and Computer Modelling 52 (2010) 215-226.

[4] S.H. Saker, Oscillation criteria of certain class of third-order nonlinear delay differential equations,Math. Slovaca 56 (2006) 433-450.

[5] J. Dzurina, I.P. Stavroulakis, Oscillation criteria for second-order delay differential equations, Appl. Math. Comput. 140 (2003) 445-453.

[6] Jiu-Gang Dong, Oscillation behavior of second order nonlinear neutral differential equations with deviating arguments, Computers and Mathematics with Applications 59 (2010) 3710-3717.

[7] T. Kusano, Y. Naito, Oscillation and nonoscillation criteria for second order quasilinear differential equations, Acta Math. Hungar. 76 (1997) 81-99.

[8] ] G.S. Ladde, V. Lakshmikantham, B.G. Zhang, Oscillation Theory of Differential Equations with Deviating Arguments, Marcel Dekker, New York, 1987.

[9] L. Liu, Y. Bai, New oscillation criteria for second-order nonlinear neutral delay differential equations, J. Comput. Appl. Math. 231 (2009) 657-663.

[10] S.H. Saker, Oscillation criteria of certain class of third-order nonlinear delay differential equations, Math. Slovaca 56 (2006) 433-450.

[11] S. H. Saker; J. Dzurina, On the oscillation of certain class of third-order nonlinear delay differential equations, Mathematica Bohemica, Vol. 135 (2010), No. 3, 225-237.

[12] R.A.sallam, New Oscillation Criteria for Second Order Nonlinear Delay Differential Equations, International Journal of Nonlinear Science ,Vol.12(2011) No.1,pp.3-11. 
[13] Samir Saker , Oscillation Theory of Delay Differential and Difference Equations 'Second and Third Orders 'VDM Verlag Dr. Muller Aktiengesellschaft \& Co. KG Dudweiler Landstr, Germany(2010) .

[14] R. Xu, F. Meng, Some new oscillation criteria for second order quasilinear neutral delay differential equations, Appl. Math. Comput. 182 (2006) 797-803.

[15] R. Xu, F. Meng, Oscillation criteria for second order quasi-linear neutral delay differential equations, Appl. Math. Comput. 192 (2007) 216-222. 